\title{
On obstacle numbers
}

\author{
Vida Dujmović* \\ Department of Computer Science and Electrical Engineering \\ University of Ottawa \\ Ottawa, Canada \\ vida.dujmovic@uottawa.ca \\ Pat Morin ${ }^{\dagger}$ \\ School of Computer Science \\ Carleton University \\ Ottawa, Canada \\ morin@scs.carleton.ca
}

Submitted: May 14, 2014; Accepted: Jun 10, 2015; Published: Jul 1, 2015

Mathematics Subject Classifications: 05C35, 05C62

\begin{abstract}
The obstacle number is a new graph parameter introduced by Alpert, Koch, and Laison (2010). Mukkamala et al. (2012) show that there exist graphs with $n$ vertices having obstacle number in $\Omega(n / \log n)$. In this note, we up this lower bound to $\Omega\left(n /(\log \log n)^{2}\right)$. Our proof makes use of an upper bound of Mukkamala et al. on the number of graphs having obstacle number at most $h$ in such a way that any subsequent improvements to their upper bound will improve our lower bound.
\end{abstract}

\section{Introduction}

The obstacle number is a new graph parameter introduced by Alpert, Koch, and Laison [2]. Let $G=(V, E)$ be a graph, let $\varphi: V \rightarrow \mathbb{R}^{2}$ be a one-to-one mapping of the vertices of $G$ onto $\mathbb{R}^{2}$ (hereafter called a drawing of $G$ ), and let $S$ be a set of connected subsets of $\mathbb{R}^{2}$. The pair $(\varphi, S)$ is an obstacle representation of $G$ when, for every pair of vertices $u, w \in V$, the edge $u w$ is in $E$ if and only if the closed line segment with endpoints $\varphi(u)$ and $\varphi(w)$ does not intersect any obstacle in $S$. An obstacle representation $(\varphi, S)$ is an

*Supported by NSERC and MRI.

${ }^{\dagger}$ Supported by NSERC. 
$h$-obstacle representation if $|S|=h$. The obstacle number of a graph $G$, denoted by $\operatorname{obs}(G)$, is the minimum value of $h$ such that $G$ has an $h$-obstacle representation. ${ }^{1}$

Note that obstacle representations of planar graphs using few obstacles often require drawings of those graphs that are far from crossing free. For example, any crossing-free drawing of the $5 \times 5$ grid, $G_{5 \times 5}$ shown in the left part of Figure 1 requires at least one obstacle in each of the sixteen internal faces (each of which has at least four sides).

It is somewhat surprising, therefore, that $G_{5 \times 5}$ has obstacle number 1 . The obstacle representation, illustrated on the right part of Figure 1 was given to us by Fabrizio Frati. In this figure, the single obstacle is drawn as a shaded region. Since at least one obstacle is clearly necessary to represent any graph other than a complete graph, this proves that $\operatorname{obs}\left(G_{5 \times 5}\right)=1$. (A similar drawing can be used to show that the $a \times b$, grid graph has obstacle number 1 , for any integers $a, b>1$.)



Figure 1: The $5 \times 5$ grid graph has obstacle number 1 .

Since their introduction, obstacle numbers have generated significant research interest $[4,5,6,7,8,9,10]$. A fundamental-and far from answered-question about obstacle numbers is that of determining the worst-case obstacle number,

$$
\operatorname{obs}(n)=\max \{\operatorname{obs}(G): G \text { is a graph with } n \text { vertices }\},
$$

of a graph with $n$ vertices.

For a graph $G=(V, E)$, we call elements of $\left(\begin{array}{c}V \\ 2\end{array}\right) \backslash E$ non-edges of $G$. The worst-case obstacle number obs $(n)$ is obviously upper bounded by $\left(\begin{array}{l}n \\ 2\end{array}\right) \in O\left(n^{2}\right)$ since, by mapping the vertices of $G$ onto a point set in sufficiently general position, one can place a small obstacle - even a single point - on the mid-point of each non-edge of $G$. No upper bound on $\operatorname{obs}(n)$ that is asymptotically better than $O\left(n^{2}\right)$ is known.

More is known about lower bounds on $\operatorname{obs}(n)$. Alpert, Koch, and Laison [2] initially show that the worst-case obstacle number is $\Omega(\sqrt{\log n / \log \log n})$ and posed as an open problem the question of determining if $\operatorname{obs}(n) \in \Omega(n)$. Mukkamala et al. [7] showed that $\operatorname{obs}(n) \in \Omega\left(n / \log ^{2} n\right)$ and Mukkamala et al. [6] later increased this to obs $(n) \in$

\footnotetext{
${ }^{1}$ Note that this definition of obstacle representation is more generous than that of Alpert, Koch, and Laison [2], which requires that the obstacles be polygonal and that the set of points determined by vertices of the obstacles and the image of $\varphi$ not contain 3 collinear points. Since the current paper proves a lower bound on the obstacle number, this lower bound also applies to the original definition.
} 
$\Omega(n / \log n)$. In the current paper, we up the lower bound again by proving the following theorem:

Theorem 1. For every integer $n>0, \operatorname{obs}(n) \in \Omega\left(n /(\log \log n)^{2}\right)$, that is, there exists a sequence, $\left\langle G_{n}\right\rangle_{n=1}^{\infty}$, such that $G_{n}$ is a graph with $n$ vertices and such that $\operatorname{obs}(G) \in$ $\Omega\left(n /(\log \log n)^{2}\right)$.

The proof of Theorem 1 makes use of an upper bound of Mukkamala et al. [6, Theorem 1] on the number of graphs having obstacle number at most $h$ in such a way that any subsequent improvements on their upper bound will result in an improved lower bound on $\operatorname{obs}(n)$.

Although some aspects of our proof are a little technical, the main idea is quite simple: Mukkamala et al. [6] show that, with probability at least $1-2^{-\Omega\left(n^{2}\right)}$, a random graph on $n$ vertices has obstacle number at least $\Omega\left(n /(\log n)^{2}\right)$. Our proof trades off a lower probability for a higher obstacle number. When all is said and done, our proof shows that, with probability at least $1-2^{-\Omega(n \log n)}$, a random graph on $n$ vertices has obstacle number at least $\Omega\left(n /(\log \log n)^{2}\right)$.

\section{The Proof}

Our proof strategy is an application of the probabilistic method [1]. We fix an arbitrary ordering, $\pi$, on the vertices of an Erdös-Rényi random graph, $G=G_{n, \frac{1}{2}}$. We then show that it is very unlikely that there is an obstacle representation, $(\varphi, S)$ of $G$ such that $|S| \in o\left(n /(\log \log n)^{2}\right)$ and the lexicographic ordering of the points assigned to vertices by $\varphi$ agrees with the ordering given by $\pi$. Here, "very unlikely" means that this occurs with probability $p<1 / n$ !. Since there are only $n$ ! possible orderings of $G^{\prime}$ s vertices, we then apply the union bound which shows that with probability $1-p n !>0$, there is no obstacle representation of $G$ using $o\left(n /(\log \log n)^{2}\right)$ obstacles, that is, obs $(G) \in \Omega\left(n /(\log \log n)^{2}\right)$.

\subsection{A Random Graph with a Fixed Ordering}

We make use of the following theorem, due to Mukkamala, Pach, and Pálvölgyi [6, Theorem 1] about the number of $n$-vertex graphs with obstacle number at most $h$ :

Theorem 2 (Mukkamala, Pach, and Pálvölgyi 2012). For any $h \geqslant 1$, the number of graphs having $n$ vertices and obstacle number at most $h$ is at most $2^{O\left(h n \log ^{2} n\right)}$.

Recall that an Erdös-Rényi random graph $G_{n, \frac{1}{2}}$ is a graph with $n$ vertices and each pair of vertices is chosen as an edge or non-edge with equal probability and independently of every other pair of vertices [3]. The following lemma shows that, for random graphs, a fixed drawing is very unlikely to yield an obstacle representation with few obstacles. Recall that the lexicographic ordering, $\prec$, for points in the plane is defined as

$$
\left(x_{1}, y_{1}\right) \prec\left(x_{2}, y_{2}\right) \text { iff } x_{1}<x_{2} \text { or }\left(x_{1}=x_{2} \text { and } y_{1}<y_{2}\right) \text {. }
$$


Lemma 1. Let $G=(V, E)$ be an Erdös-Rényi random graph $G_{n, \frac{1}{2}}$, let $v_{1}, \ldots, v_{n}$ be an ordering of the vertices in $V$ that is independent of the choices of edges in $G$, and let $(\varphi, S)$ be an obstacle representation of $G$ using the minimum number of obstacles subject to the constraint that

$$
\varphi\left(v_{1}\right) \prec \varphi\left(v_{2}\right) \prec \cdots \varphi\left(v_{n}\right),
$$

where $\prec$ denotes the lexicographic ordering of points. Then, for any constant $c>0$,

$$
\operatorname{Pr}\left\{|S| \in \Omega\left(n /(\log \log n)^{2}\right)\right\} \geqslant 1-e^{-c n \log n} .
$$

Proof. Fix some integer $k=k(n) \in \omega_{n}(1)$ to be specified later and first consider the subgraph $G_{0}$ of $G$ induced by the vertices $v_{1}, \ldots, v_{k}$. Applying Theorem 2 with $n=k$ and $h=\alpha k / \log ^{2} k$, we obtain

$$
\operatorname{Pr}\left\{\operatorname{obs}\left(G_{0}\right) \leqslant \alpha k / \log ^{2} k\right\} \leqslant \frac{2^{O\left(\alpha k^{2}\right)}}{2^{\left(\begin{array}{c}
k \\
2
\end{array}\right)}} \leqslant e^{-\beta k^{2}},
$$

where $\beta>0$ for a sufficiently small constant $\alpha>0$, and sufficiently large $k$. Note that, if $\operatorname{obs}\left(G_{0}\right) \geqslant h$, then, in the obstacle representation $(\varphi, S)$, there must be at least $h-1$ obstacles of $S$ that are contained in the convex hull of $\varphi\left(v_{1}\right), \ldots, \varphi\left(v_{k}\right)$; this is because the obstacle representation $(\varphi, S)$ can be turned into an obstacle representation of $G_{0}$ by merging all obstacles that are not contained in the convex hull of $\varphi\left(v_{1}\right), \ldots, \varphi\left(v_{k}\right)$.

Let $m=\lfloor n / k\rfloor$ and notice that the preceding argument applies to any subset $V_{i}=$ $\left\{v_{k i+1}, \ldots, v_{(k+1) i}\right\}$ of vertices, for any $i \in\{0, \ldots, m-1\}$. That is, Equation (1) shows that, with probability at least $1-2^{-\Omega\left(k^{2}\right)}$, the obstacle number of the subgraph $G_{i}$ induced by $V_{i}$ is $\Omega\left(k / \log ^{2} k\right)$. If this occurs, then $S$ has $\Omega\left(k / \log ^{2} k\right)$ obstacles that are completely contained in the convex hull of $V_{i}$. In particular, the obstacles contained in the convex hull of $V_{i}$ are different from the obstacles contained in the convex hull of $V_{j}$, for all $j \neq i$.

We are proving a lower bound on the number of obstacles, so we are worried about the case where the number of convex hulls that do not contain at least $\alpha k / \log ^{2} k$ obstacles exceeds $m / e .^{2}$ The number of convex hulls, $M$, not containing at least $\alpha k / \log ^{2} k$ obstacles is dominated by a $\operatorname{binomial}\left(m, e^{-\beta k^{2}}\right)$ random variable. Using Chernoff's bound on the tail of a binomial random variable, ${ }^{3}$ we have that

$$
\begin{aligned}
\operatorname{Pr}\{M \geqslant m / e\} & \left.=\operatorname{Pr}\{M \geqslant(1+\delta) \mu\} \quad \text { (where } \mu=m e^{-\beta k^{2}} \text { and } \delta=e^{\beta k^{2}-1}-1\right) \\
& \leqslant\left(\frac{e^{\delta}}{(1+\delta)^{1+\delta}}\right)^{\mu} \\
& \leqslant\left(\frac{e^{e^{\beta k^{2}}}}{\left(e^{\beta k^{2}-1}\right)^{e^{\beta k^{2}-1}}}\right)^{m e^{-\beta k^{2}}}
\end{aligned}
$$

${ }^{2}$ Euler's constant $e=\lim _{n \rightarrow \infty}(1-1 / n)^{n}$ is just a convenient constant to use here.

${ }^{3}$ Chernoff's Bound: For any binomial $(m, p)$ random variable, $B$, any $\delta>0$ and $\mu=m p$,

$$
\operatorname{Pr}\{B \geqslant(1+\delta) \mu\} \leqslant\left(\frac{e^{\delta}}{(1+\delta)^{1+\delta}}\right)^{\mu} .
$$




$$
\begin{aligned}
& =\left(\frac{e^{e^{\beta k^{2}}}}{e^{\left(\beta k^{2}-1\right) e^{\beta k^{2}-1}}}\right)^{m e^{-\beta k^{2}}} \\
& =\frac{e^{m}}{e^{m\left(\beta k^{2}-1\right) e^{\beta k^{2}-1} e^{-\beta k^{2}}}} \\
& =\frac{e^{m}}{e^{m\left(\beta k^{2}-1\right) / e}} \\
& =e^{-\Omega\left(m k^{2}\right)}
\end{aligned}
$$

Taking $k=c^{\prime} \log n$, for a sufficiently large constant, $c^{\prime}$, and recalling that $m=\lfloor n / k\rfloor$, we obtain the desired result. In particular,

$$
|S| \geqslant \Omega\left(\left(k / \log ^{2} k\right) \cdot(m-m / e)\right)=\Omega\left(n /(\log \log n)^{2}\right)
$$

with probability at least

$$
1-e^{-\Omega\left(m k^{2}\right)}=1-e^{-\Omega\left(c^{\prime} n \log n\right)} \geqslant 1-e^{-c n \log n},
$$

for all $n$ greater than some sufficiently large constant $n_{0}$. For $n \in\left\{1, \ldots, n_{0}\right\}$, the lemma is trivially satisfied since $|S| \geqslant 0$ with probability $1 \geqslant 1-e^{-c n \log n}$.

\section{$2.2 \quad$ Finishing Up}

For completeness, we now spell out the proof of Theorem 1.

Proof of Theorem 1. Let $G=(V, E)$ be an Erdös-Rényi random graph with $n$ vertices with vertex set $V=\{1, \ldots, n\}$. For every obstacle representation $(\varphi, S)$ of $G$, there is an ordering on $V$ given by the lexicographic ordering of the points $\{\varphi(v): v \in V\}$.

By Lemma 1, the probability that a particular such ordering, $v_{1}, \ldots, v_{n}$, allows an obstacle representation using $o\left(n /(\log \log n)^{2}\right)$ obstacles is at most $p \leqslant e^{-c n \log n}$ for every constant $c>0$. In particular, for sufficiently large $c$, we have $p<1 / n$ !. By the union bound the probability that there is any ordering that supports an obstacle representation of $G$ with $o\left(n /(\log \log n)^{2}\right)$ obstacles is at most

$$
\hat{p}=p \cdot n !<1
$$

We deduce that there exists some graph, $G^{\prime}$, with $\operatorname{obs}\left(G^{\prime}\right) \in \Omega\left(n /(\log \log n)^{2}\right)$.

\section{Remarks}

Our proof of Theorem 1 relates the problem of counting the number of $n$-vertex graphs with obstacle number at most $h$ to the problem of determining the worst-case obstacle number of a graph with $n$ vertices. Currently, we use Theorem 2 of Mukkamala et al. [7], which proves an upper bound of $e^{O\left(h n \log ^{2} n\right)}$ on the number of $n$-vertex graphs with obstacle number at most $h$. 
Any improvement on the upper bound for the counting problem will immediately translate into an improved lower bound on the worst-case obstacle number. Let $f(h, k)$ denote the number of $k$-vertex graphs with obstacle number at most $h$ and let

$$
\hat{h}(k)=\max \left\{h: f(h, k) \leqslant 2^{k^{2} / 4}\right\} .
$$

The quantity $\hat{h}(k)$ is chosen so that a random graph on $k$ vertices has probability at most $2^{-\Omega\left(k^{2}\right)}$ of having obstacle number less than $\hat{h}(k)$; Theorem 2 shows that $\hat{h}(k) \in$ $\Omega\left(k /(\log k)^{2}\right)$. Our proof of Lemma 1 shows that there exist graphs with obstacle number at least $\Omega(n \hat{h}(c \log n) /(c \log n))$.

We note that our technique gives an improved lower bound until someone is able to prove that $\hat{h}(n) \in \Omega(n)$. At this point, our approach gives a lower bound worse than the trivial lower bound $\hat{h}(n)$.

We conjecture that improved upper bounds on $f(h, n)$ that reduce the dependence on $h$ are the way forward:

Conjecture 1. $f(h, n) \leqslant 2^{g(n) \cdot o(h)}$, where $g(n) \in O\left(n \log ^{2} n\right)$.

In support of this conjecture, we observe that an upper bound of the form $f(1, n) \leqslant$ $2^{g(n)}$ is sufficient to give the crude upper bound $f(h, n) \leqslant 2^{h \cdot g(n)}$ since any graph with an $h$-obstacle representation is the common intersection of $h$ graphs that each have a 1-obstacle representation. That is, if obs $(G) \leqslant h$, then there exists $E_{1}, \ldots, E_{h}$ such that $G=\left(V, \bigcap_{i=1}^{h} E_{i}\right)$ and $\operatorname{obs}\left(V, E_{i}\right)=1$ for all $i \in\{1, \ldots, h\}$. This seems like a very crude upper bound in which many graphs are counted multiple times. Conjecture 1 asserts that this argument overestimates the dependence on $h$.

\section{Acknowledgements}

This work was initiated at the Workshop on Geometry and Graphs, held at the Bellairs Research Institute, March 10-15, 2013. We are grateful to the other workshop participants for providing a stimulating research environment.

A previous draft of this article proved a version Lemma 1 for a fixed drawing, $\varphi$, and then went to great lengths to argue that the number of combinatorially distinct drawings was at most $2^{O(n \log n)}$. We are grateful to an anonymous referee who pointed out that the proof of Lemma 1 also holds when only the lexicographic ordering of the vertices is fixed, thereby eliminating the need to bound the number of combinatorially equivalent drawings.

\section{References}

[1] N. Alon and J. H. Spencer. The Probabilistic Method. John Wiley \& Sons, Hoboken, third edition, 2008.

[2] H. Alpert, C. Koch, and J. D. Laison. Obstacle numbers of graphs. Discrete \&6 Computational Geometry, 44(1):223-244, 2010. 
[3] P. Erdős and A. Rényi. On random graphs. Publicationes Mathematicae, 6:290-297, 1959.

[4] R. Fulek, N. Saeedi, and D. Sariöz. Convex obstacle numbers of outerplanar graphs and bipartite permutation graphs. In J. Pach, editor, Thirty Essays on Geometric Graph Theory, pages 249-261. Springer, New York, 2013.

[5] M. P. Johnson and D. Sariöz. Computing the obstacle number of a plane graph, 2011. arXiv:1107.4624

[6] P. Mukkamala, J. Pach, and D. Pálvölgyi. Lower bounds on the obstacle number of graphs. Electr. J. Comb., 19(2):\#P32, 2012.

[7] P. Mukkamala, J. Pach, and D. Sariöz. Graphs with large obstacle numbers. In D. M. Thilikos, editor, WG, volume 6410 of Lecture Notes in Computer Science, pages 292-303, 2010.

[8] J. Pach and D. Sariöz. Small $(2, s)$-colorable graphs without 1-obstacle representations, 2010. arXiv:1012.5907

[9] J. Pach and D. Sariöz. On the structure of graphs with low obstacle number. Graphs and Combinatorics, 27(3):465-473, 2011.

[10] D. Sariöz. Approximating the obstacle number for a graph drawing efficiently. In Proceedings of the 23rd Canadian Conference on Computational Geometry (CCCG 2011), 2011. 